\title{
Suppression of Rayleigh-Taylor turbulence by time-periodic acceleration
}

\author{
G. Boffetta \\ Dipartimento di Fisica and INFN, Università di Torino, via P. Giuria 1, 10125 Torino, Italy \\ M. Magnani \\ Dipartimento di Fisica, Università di Torino, via P. Giuria 1, 10125 Torino, Italy \\ S. Musacchio \\ Université Côte d'Azur, CNRS, LJAD, Nice 06108, France
}

(Received 10 July 2018; revised manuscript received 1 February 2019; published 11 March 2019)

\begin{abstract}
The dynamics of Rayleigh-Taylor turbulence convection in the presence of an alternating, time-periodic acceleration is studied by means of extensive direct numerical simulations of the Boussinesq equations. Within this framework, we discover a mechanism of relaminarization of turbulence: the alternating acceleration, which initially produces a growing turbulent mixing layer, at longer times suppresses turbulent fluctuation and drives the system toward an asymptotic stationary configuration. Dimensional arguments and linear stability theory are used to predict the width of the mixing layer in the asymptotic state as a function of the period of the acceleration.
\end{abstract}

DOI: 10.1103/PhysRevE.99.033110

\section{INTRODUCTION}

It is well known that it is possible to stabilize an unstable fixed point of a mechanical system by a periodic modulation of the forces. A famous example is the Kapitza inverted pendulum: the upper fixed point may became stable when the suspension point is subject to fast oscillations [1]. The dynamic stabilization has been extended to fluid dynamics with the experimental evidence that the Rayleigh-Taylor (RT) unstable configuration of superimposed liquids of different densities [2-5] can be stabilized by oscillating the liquids perpendicular to the horizontal interface [6]. Stabilization in this case requires a minimal viscosity of one of the liquids, which prevents the development of parametric resonances at high frequencies [7]. The possibility to control RT instability is important because it has many applications, from confined nuclear fusion [8,9] to plasma physics [9] and laser matter interactions [10].

In the standard case of constant acceleration, the unstable interface evolves into a turbulent mixing layer that broadens in time with a well-characterized phenomenology $[11,12]$. However, there are important applications where acceleration changes in time and may even reverse sign. Examples include inertial confined fusion [13,14], pulsating stars, and supernovae explosions [15]. RT instability and turbulence with complex acceleration history have been studied experimentally [16] and numerically [17-19]. In particular, the effects of a single stabilizing phase (corresponding to gravity reversal) have been shown to produce a slowing down of the turbulent mixing layer [16] and an increase of small scale mixing [20].

In this paper, we address the question of what happens to RT turbulence in the presence of time-periodic acceleration that continuously alternates phases of unstable and stable stratification. By extensive numerical simulations of the Boussinesq model, we find that it is possible to restabilize the turbulent phase and that the turbulent mixing layer, which initially develops and grows quadratically in time, eventually stops for any period of the acceleration modulation. The mechanism at the basis of this surprising suppression of RT mixing is the decay of the turbulent fluctuations during the stable phase, with a reduction of the temperature and density fluctuations in the mixing layer. We find simple scaling laws for the arresting time and the asymptotic size of the mixing layer, and we show how the suppression of turbulence can be understood in terms of linear stability theory.

\section{FORMULATION OF THE MODEL}

We consider the Boussinesq model of an incompressible velocity field $\mathbf{u}(\mathbf{x}, t)$ coupled with a temperature (density) field $\theta(\mathbf{x}, t)$ in the presence of a time-dependent acceleration $\mathbf{g}(t)=-g(t) \hat{\mathbf{z}}$ in the vertical direction,

$$
\begin{aligned}
& \frac{\partial \mathbf{u}}{\partial t}+\mathbf{u} \cdot \nabla \mathbf{u}=-\nabla p+v \nabla^{2} \mathbf{u}-\beta \mathbf{g} \theta, \\
& \frac{\partial \theta}{\partial t}+\mathbf{u} \cdot \nabla \theta=\kappa \nabla^{2} \theta,
\end{aligned}
$$

where $v$ is the kinematic viscosity, $\kappa$ is the thermal diffusivity, and $\beta$ is the thermal expansion coefficient. We assume standard initial conditions for RT, with $\mathbf{u}(\mathbf{x}, t)=0$ and $\theta(\mathbf{x}, 0)=$ $-\left(\theta_{0} / 2\right) \operatorname{sgn}(z)$, where $\theta_{0}$ is the temperature jump that defines the Atwood number $A=\beta \theta_{0} / 2$.

In the case of constant $g$, the usual phenomenology of RT turbulence is expected. The initial condition is unstable with respect to perturbation of the interface $z=0$ and, for a singlemode perturbation at wave number $k$, the linear stability analysis for an inviscid potential flow gives the growth rate of the amplitude $\lambda(k)=\sqrt{A g k}$, while the high wave numbers are stabilized by viscosity and diffusivity [5,21]. After this linear phase, the system enters a nonlinear regime in which a 
turbulent mixing layer grows starting from the interface at $z=$ 0 . Within the mixing layer, potential energy is converted to turbulent kinetic energy, with large-scale velocity fluctuations increasing dimensionally as $u_{\mathrm{rms}} \simeq A g t$. Consequently, the width of the mixing layer $h$ grows as $h(t) \simeq A g t^{2}$ [12]

As discussed, in this paper we consider the case of periodic acceleration $g(t)$. To be specific, we study the case of a square wave of amplitude $g_{0}$ in which the sign of $g(t)$ is reversed every half-period $T / 2$ (starting from the positive, unstable value). We also studied the different protocol of sinusoidal function $g(t)=g_{0} \cos (2 \pi t / T)$ (not reported here) to verify the generality of our results.

We performed extensive numerical simulations of the Boussinesq equations (1) and (2) by means of fully dealiased pseudospectral code in a three-dimensional (3D) box of dimension $L_{x}=L_{y}=L_{z} / 4$ at resolution $M \times M \times 4 M$ for two values $M=256$ and 512 with uniform grid [22]. The boundary conditions for velocity are periodic in the lateral directions and no-slip in the vertical direction. Time evolution is obtained by a second-order Runge-Kutta scheme with an explicit linear part. The code is fully parallelized in one direction by using MPI libraries. For all runs we fix $\operatorname{Pr}=\nu / \kappa=1$ and viscosity is sufficiently large to resolve the small scales during all the phases of the simulations $\left(\nu=5 \times 10^{-4}\right.$ for $M=256$ and $v=3 \times 10^{-4}$ for $M=512$ ) [23]. In all runs, the value of the period $T$ is taken sufficiently long to allow for the development of the turbulent mixing layer before inverting the acceleration, i.e., $\lambda(k) T>1$. Numerical results are made dimensionless by using $L_{x}, \tau=\sqrt{L_{x} /\left(A g_{0}\right)}$, and $\theta_{0}$ as the unit length, time, and temperature, respectively. The typical Reynolds number reached by the flow (defined in terms of the vertical velocity fluctuations and on the width of the mixing layer) is about $2 \times 10^{4}$.

RT instability is seeded by perturbing the initial temperature field with respect to the unstable step profile. Two different perturbations were implemented. In the first case, we perturbed the initial condition by adding $5 \%$ of white noise to the value of $\theta(\mathbf{x}, 0)$ in a thin layer of width $L_{z} / 512$ around the interface $z=0$. In the second set of simulations, we perturb the interface by a superposition of $2 \mathrm{D}$ linear waves of small amplitudes and a narrow range of wave numbers around $k=30$ with a flat spectrum, similar to the AS-0 perturbation in [19]. The two sets of simulations gave similar results for long-time statistics. The results reported in this paper are relative to the first type of perturbation, and they are averaged over 10 independent realizations of the white noise for each value of the parameters.

\section{NUMERICAL RESULTS}

Figure 1 shows the vertical sections of the temperature field for the simulation at period $T=3 \tau$ at different times. At $t=1.5 T$ the system starts to develop the turbulent mixing layer around the interface, and in the usual case of constant acceleration. After four periods of acceleration (central panel), the mixing layer has grown to reach about one-half of the domain. After 10 periods (right panel) we observe that the mixing layer has almost the same width and has become more homogeneous in the horizontal direction due to the decay of turbulent fluctuations.

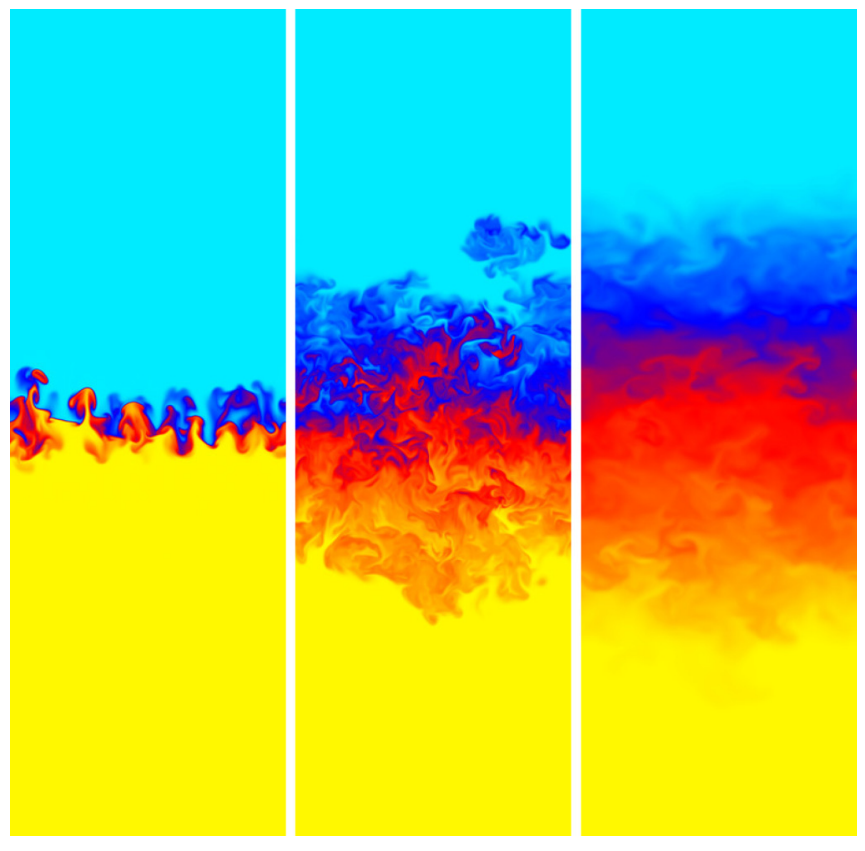

FIG. 1. Vertical sections of size $L_{x} \times L_{z}$ of the temperature field (yellow hot, blue cold) for Rayleigh-Taylor turbulence in periodic acceleration of period $T=3 \tau$ at times $t=1.5 T$ (left), $t=4 T$ (center), and $t=10 T$ (right). Simulation at resolution $M=512$.

Figure 2 shows the time evolution of the vertical rms velocity for a simulation with period $T=\tau$. At the beginning we observe a growth of $w_{\text {rms }}$ with large oscillations, which reflects the presence of accelerating and decelerating phases. Nonetheless, after about four periods the amplitude of the oscillations reduces and vertical velocity fluctuations decay asymptotically. In the inset we plot the same quantity at short time for the case $T=3 \tau$, compared with the periodicity of the acceleration. During the unstable phases (gray regions) there is on average a growth of the velocity fluctuations, initially in agreement with the dimensional linear law. After the first periods, the growth of $w_{\mathrm{rms}}$ is preceded by a transient

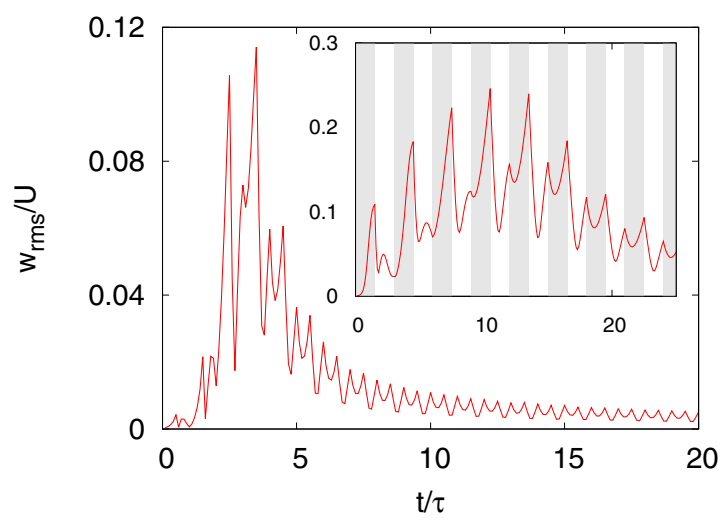

FIG. 2. rms of the vertical velocity component in the mixing layer, made dimensionless with $U=A g_{0} \tau$, as a function of dimensionless time for the case of period $T=\tau$. Inset: the same quantity for the case of period $T=3 \tau$ with gray (white) regions representing the unstable (stable) phase. Simulations with $M=512$. 

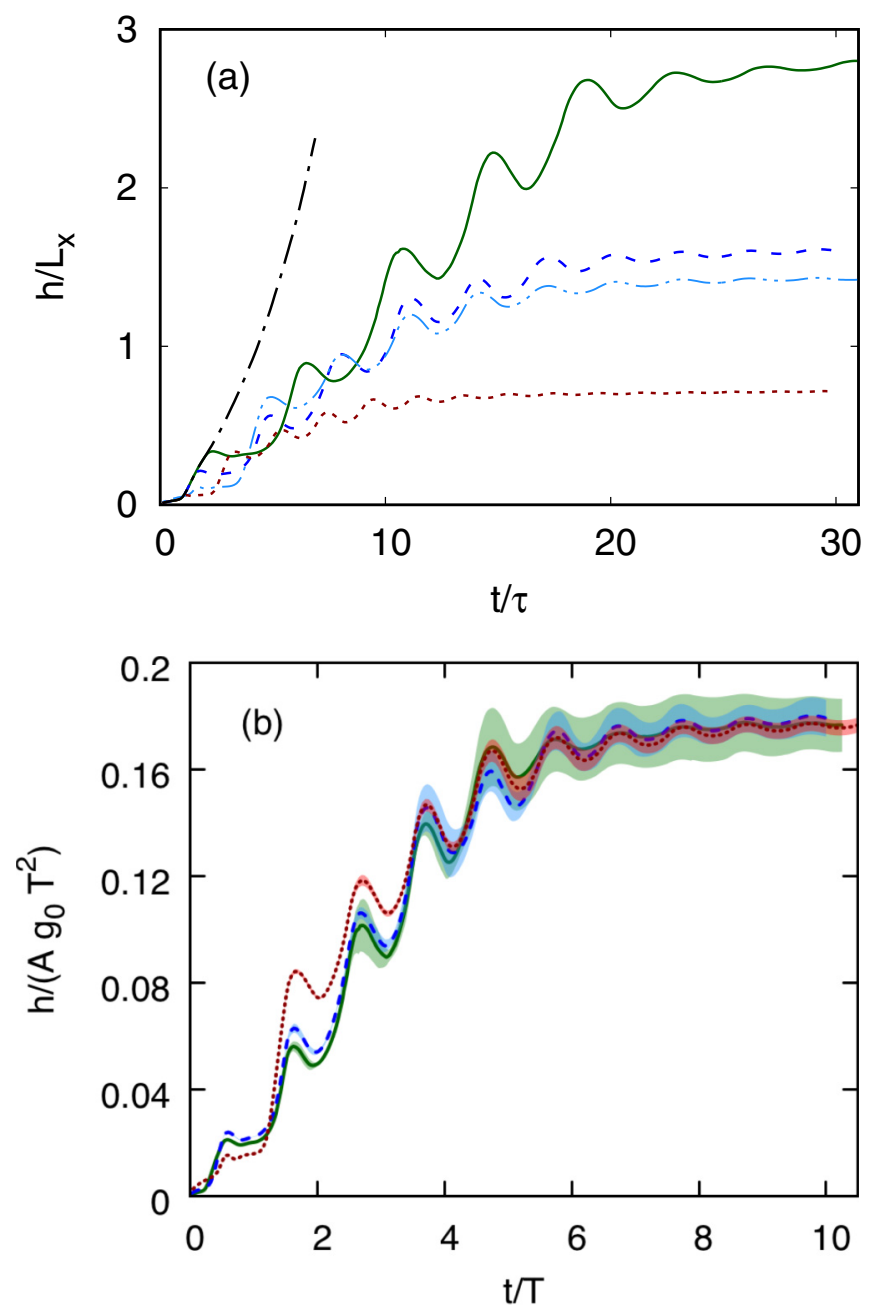

FIG. 3. (a) Evolution of the width of the mixing layer for four simulations at resolution $M=512$ starting from the same set of initial conditions with different periods of the acceleration: $T=\infty$ (black dot-dashed line), $T=2 \tau$ (red dotted line), $T=3 \tau$ (blue dashed line), and $T=4 \tau$ (green continuous line). The dot-dotdashed light blue line represents the case $T=3 \tau$ for resolution $M=256$ with larger viscosity. (b) The same data plotted by rescaling time with the period $T$ and space with $A g_{0} T^{2}$, with ensemble rms (shadow area).

decrease. This is due to the fact that, immediately after each acceleration reversal, the plumes must invert the direction of their motion before accelerating again. The inset of Fig. 2 also shows a secondary peak of vertical velocity in the stable phases (white regions), which is caused by the exchange between kinetic and potential energy in stably stratified turbulence. The period of these secondary oscillations $T_{\mathrm{BV}}=$ $2 \pi / N$ is determined by the Brunt-Väisälä frequency $N=$ $\sqrt{g_{0} \beta|\partial\langle\theta\rangle / \partial z|}$, where $\langle\theta(z, t)\rangle \equiv 1 /\left(L_{x} L_{y}\right) \int d x \int d y \theta(\mathbf{x}, t)$ is the mean temperature profile. The initial strong gradient of temperature at the interface gives large values of $N$ and therefore a short secondary period $T_{\mathrm{BV}} \ll T$. By approximating the mean temperature gradient as $|\partial\langle\theta\rangle / \partial z| \simeq \theta_{0} / h$, we obtain $N \simeq \sqrt{2 A g_{0} / h}$. Therefore, the growth of the mixing layer causes a decrease of the Brunt-Väisälä frequency.
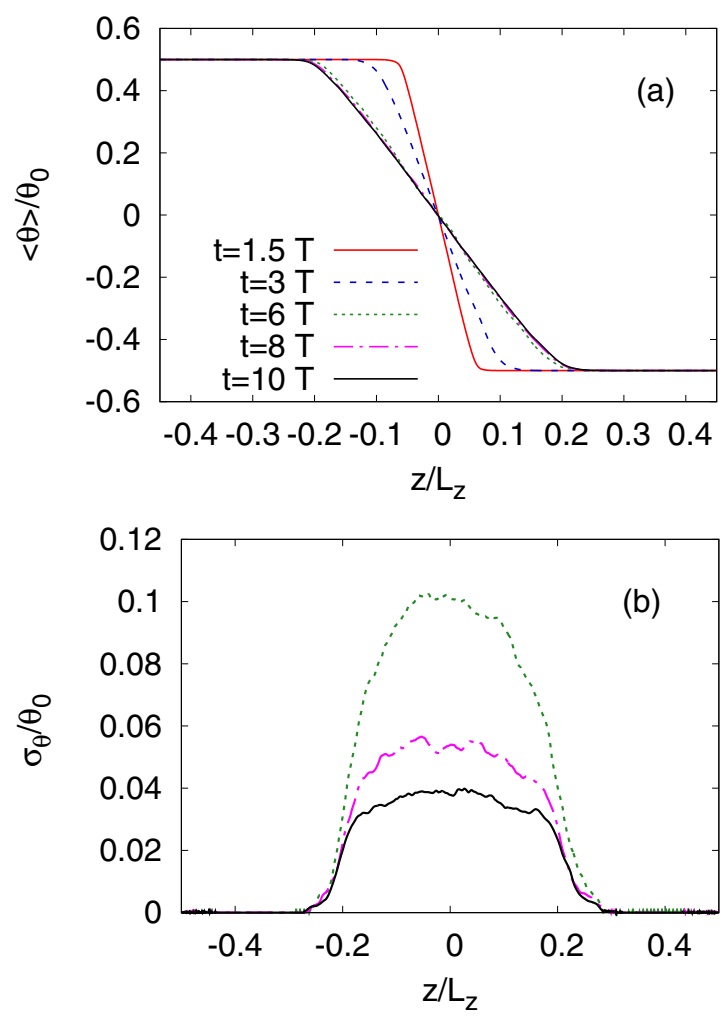

FIG. 4. (a) Mean temperature profile $\langle\theta\rangle$ for the simulation with period $T=3 \tau$ at times $t=1.5 T$ (red continuous line), $t=3 T$ (blue dashed line), $t=6 T$ (green dotted line), $t=8 T$ (magenta dot-dashed line), and $t=10 T$ (black full line). (b) Temperature standard deviation $\sigma$ for the simulation with period $T=3 \tau$ at times $t=6 T$ (green dotted line), $t=8 T$ (magenta dot-dashed line), and $t=10 T$ (black full line). Simulations with $M=512$.

The long-time decay of velocity fluctuations indicates that asymptotically the unstable phase is unable to sustain turbulence in the mixing layer, which is eventually arrested. This surprising result is indeed observed in our simulations, as shown qualitatively in Fig. 1. More quantitatively, in Fig. 3 we show the time evolution of the width $h(t)$ of the mixing layer, which is defined from the mean temperature profiles as the difference between the two heights $z_{ \pm}$at which $\left\langle\theta\left(z_{ \pm}, t\right)\right\rangle=$ $\pm 0.95\left(\theta_{0} / 2\right)$. In all the cases investigated, after a few oscillations the width of the mixing layer reaches an asymptotic value $h_{\infty}$. Strictly speaking, a truly asymptotic value cannot be reached because of the presence of a diffusive term in (2), but since the value of $\kappa$ in our simulations is very small, its effect is observable only on much longer timescales.

The period of the accelerations is the only external timescale in the dynamics, therefore one is tempted to rescale time in Fig. 3 with $T$ and correspondingly the spatial scale with the dimensional expression $A g_{0} T^{2}$. With this rescaling we observe an almost perfect collapse of the curves $h(t)$ at different periods of oscillation. The asymptotic width of the mixing layer is found to collapse to $h_{\infty} \simeq 0.18 A g_{0} T^{2}$ and the corresponding period of the Brunt-Väisälä oscillations is $T_{\mathrm{BV}} \simeq 1.9 T$.

Figure 4 shows the mean temperature profiles $\langle\theta(z, t)\rangle$ and the temperature standard deviation $\sigma_{\theta}(z, t)=\left(\left\langle\theta^{2}\right\rangle-\langle\theta\rangle^{2}\right)^{1 / 2}$ 
at different times in the evolution of the mixing layer. As in usual RT turbulence, the average temperature $\langle\theta\rangle$ develops a mean linear profile [24] that evolves in a self-similar way until it is stopped (at $t \geqslant 8 T$; see Fig. 3 ). At late times the mean temperature profile remains frozen, while temperature fluctuations, represented by the standard deviation in Fig. 4, decay in time, following the decay of velocity fluctuations shown in Fig. 2. All together, these results show that the mechanism that initially produces turbulence is unable to sustain the flow for long times. Such a novel phenomenon of asymptotic relaminarization of turbulence within the mixing layer in the time-periodic RT system is reminiscent of the relaminarization observed in pipe flows [25].

The physical interpretation of turbulence suppression and of the observed rescaling is the following. During the stable phase, turbulence decays and velocity and temperature fluctuations are reduced, as shown in Figs. 2 and 4. When the acceleration is switched back to the unstable phase, it takes some time for the available potential energy to produce new turbulent fluctuations and associated kinetic energy [19], and this time increases with the width of the mixing layer. Although the rate of this nonlinear instability cannot be analytically computed, one can use the results for the linear instability growth rate, an approximation that is increasingly well justified with time since turbulent fluctuations are decaying. For the case of a linear temperature gradient of width $h$ that connects two constant plateaus (an idealized model of the profiles of Fig. 4), linear stability analysis predicts the growth rate $\lambda(k)$ of an inviscid perturbation at wave number $k$ as [26]

$$
\lambda^{2}(k)=\frac{A g_{0} k}{A+(1-A) h k\left[1-e^{-h k}\right]^{-1}},
$$

which recovers the standard result $\lambda^{2}=A g_{0} k$ in the limit of very steep gradient $h k \rightarrow 0$. In the opposite limit of a wide mixing layer, which is relevant for the present situation, $h k \gg$ 1 and (3) gives

$$
\lambda^{2}(k)=\frac{A g_{0}}{(1-A) h},
$$

which shows that the growth rate become independent of $k$ and decays as $1 / \sqrt{h}$. Therefore by increasing $h, 1 / \lambda$ becomes eventually longer than $T / 2$ and the perturbation does not have sufficient time to grow. From (4) this happens at a scale that is proportional to $T^{2}$, in agreement with the phenomenological rescaling used in Fig. 3. Numerical confirmation of this argument is given by the fact that by stopping the periodic acceleration reversal during the unstable condition after the asymptotic stage is reached, we observe the recovery of the quadratic growth of the mixing layer after a time longer than $T / 2$.

Since RT turbulence is known to produce an efficient exchange of heat between the two layers at different temperatures, it is interesting to investigate how this is affected by the acceleration reversal. We have therefore computed the evolution of the Rayleigh number $\mathrm{Ra}=A g_{0} h^{3} /(\nu \kappa)$ and of the Nusselt number $\mathrm{Nu}=\langle w \theta\rangle h /\left(\kappa \theta_{0}\right)$ in our numerical simulations. We remind the reader that in standard RT turbulence, both $\mathrm{Ra}$ and $\mathrm{Nu}$ grow in time (following the growth of $h$ and of turbulent velocities) and the dependence of $\mathrm{Nu}$ on $\mathrm{Ra}$ realizes

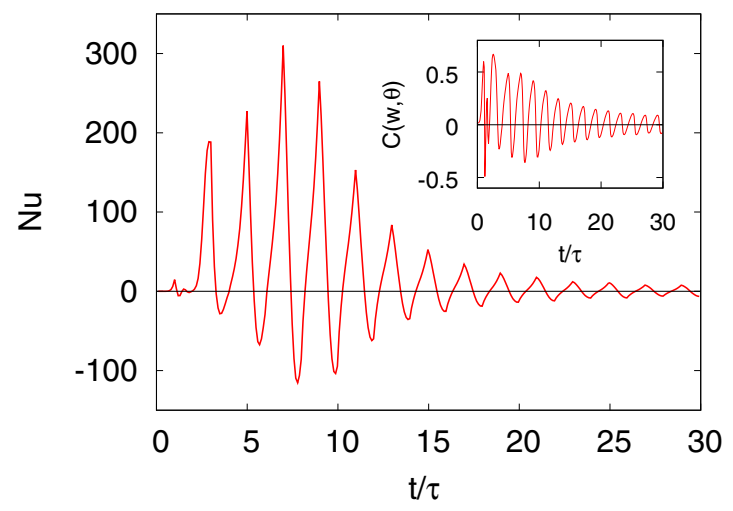

FIG. 5. Time evolution of the Nusselt number for the case $T=2 \tau$. Inset: time evolution of the correlation $C(w, \theta)=$ $\langle w \theta\rangle /\left(w_{\mathrm{rms}} \theta_{\mathrm{rms}}\right)$. Simulations with $M=512$.

the so-called "ultimate state of thermal convection" for which $\mathrm{Nu} \simeq \mathrm{Ra}^{1 / 2}$ [27-29].

In the present case, since the mixing scale $h$ is arrested, the Rayleigh number reaches an asymptotic value $\operatorname{Ra}_{\text {max }} \propto T^{6}$, while the Nusselt number, shown in Fig. 5, decays in time as a consequence of the reduction of velocity fluctuations (see Fig. 2). We observe also that $\mathrm{Nu}$ is negative during the stable phase as a consequence of the inversion of the vertical velocity, which becomes anticorrelated with temperature fluctuations. The decay of $\mathrm{Nu}$ is not simply due to the reduction of vertical velocity fluctuations: indeed also the correlation $C(w, \theta)=\langle w \theta\rangle /\left(w_{\mathrm{rms}} \theta_{\mathrm{rms}}\right)$ between the velocity and the temperature fields decreases in time, as shown in the inset of Fig. 5. We also observe an increasing symmetry between the stable and the unstable phases in the oscillations of $\mathrm{Nu}$. As a consequence, after a few periods of oscillations, the total heat flux over a period is close to zero: the positive flux during the unstable phases is compensated by an equal and opposite flux in the stable phase.

\section{CONCLUSIONS}

We have studied the phenomenology of Rayleigh-Taylor turbulence in the Boussinesq approximation in the presence of a periodic acceleration that alternates phases of unstable and stable stratification. Remarkably, we have found that after a few periods $T$ of the acceleration, the turbulent mixing layer reaches an asymptotic finite extension of amplitude proportional to $T^{2}$. In this state, turbulence is found to decay in time, in spite of the presence of phases of unstable stratification, and it becomes ineffective to develop further instabilities. Extensive numerical simulations show that this result is robust in the sense that the presence of an asymptotic layer is independent of the period $T$ (in the range of period compatible with the size of the domain) and also of the specific protocol of the acceleration (sinusoidal or square wave).

Our result extends the phenomenon of dynamic stabilization of the RT instability by fast oscillation to the fully nonlinear, turbulent case as it shows that it is possible to suppress turbulent convection by a periodic modulation of the acceleration field. Our results have been obtained within the Boussinesq approximation of an incompressible flow. It would 
be very interesting to investigate if this mechanism persists in non-Boussinesq conditions.

More generally, our result suggests possible applications beyond the specific RT configuration. For example, it is well known that parametric excited Rayleigh-Bénard convection, by thermal or acceleration fast modulation, changes the onset of the instability for convection [28,30,31]. The present work shows that also the nonlinear, turbulent phase could in principle be controlled (and suppressed) by appropriate modulation of the external acceleration. The possibility to observe this effect in other systems would be extremely interesting, encouraging further numerical or experimental investigations.

\section{ACKNOWLEDGMENTS}

G.B. acknowledges support by the project CSTO162330 Extreme Events in Turbulent Convection and from the Departments of Excellence grant (MIUR). M.M. thanks the financial support by the project CRT 2015.2697. The HPC center CINECA is gratefully acknowledged for computing resources.
[1] V. I. Arnold, Mathematical Methods of Classical Mechanics (Springer, New York, 1989).

[2] S. Chandrasekhar, Hydrodynamic and Hydromagnetic Stability (Courier, Mineola, NY, 2013).

[3] D. Sharp, Physica D 12, 3 (1984).

[4] S. Abarzhi, Philos. Trans. R. Soc. London, Ser. A 368, 1809 (2010).

[5] Y. Zhou, Phys. Rep. 720-722, 1 (2017).

[6] G. Wolf, Phys. Rev. Lett. 24, 444 (1970).

[7] F. Troyon and R. Gruber, Phys. Fluids 14, 2069 (1971).

[8] J. Kilkenny, S. Glendinning, S. Haan, B. Hammel, J. Lindl, D. Munro, B. Remington, S. Weber, J. Knauer, and C. Verdon, Phys. Plasmas 1, 1379 (1994).

[9] M. Tabak, J. Hammer, M. Glinsky, W. Kruer, S. Wilks, J. Woodworth, E. Campbell, M. Perry, and R. Mason, Phys. Plasmas 1, 1626 (1994).

[10] R. J. Taylor, J. P. Dahlburg, A. Iwase, J. H. Gardner, D. E. Fyfe, and O. Willi, Phys. Rev. Lett. 76, 1643 (1996).

[11] M. Chertkov, Phys. Rev. Lett. 91, 115001 (2003).

[12] G. Boffetta and A. Mazzino, Annu. Rev. Fluid Mech. 49, 119 (2017).

[13] S. Nakai and H. Takabe, Rep. Prog. Phys. 59, 1071 (1996).

[14] R. Betti, C. D. Zhou, K. S. Anderson, J. L. Perkins, W. Theobald, and A. A. Solodov, Phys. Rev. Lett. 98, 155001 (2007).

[15] S. A. Colgate and R. H. White, Astrophys. J. 143, 626 (1966).

[16] G. Dimonte, P. Ramaprabhu, and M. Andrews, Phys. Rev. E 76, 046313 (2007).
[17] D. Livescu, T. Wei, and M. Petersen, J. Phys.: Conf. Ser. 318, 082007 (2011).

[18] P. Ramaprabhu, V. Karkhanis, R. Banerjee, H. Varshochi, M. Khan, and A. G. W. Lawrie, Phys. Rev. E 93, 013118 (2016).

[19] D. Aslangil, A. Banerjee, and A. G. W. Lawrie, Phys. Rev. E 94, 053114 (2016).

[20] P. Ramaprabhu, V. Karkhanis, and A. G. W. Lawrie, Phys. Fluids 25, 115104 (2013).

[21] H. Kull, Phys. Rep. 206, 197 (1991).

[22] J. P. Boyd, Chebyshev and Fourier Spectral Methods (Dover, New York, 2001).

[23] G. Boffetta, A. Mazzino, S. Musacchio, and L. Vozella, Phys. Fluids 22, 035109 (2010).

[24] G. Boffetta, F. De Lillo, and S. Musacchio, Phys. Rev. Lett. 104, 034505 (2010).

[25] B. Eckhardt, T. M. Schneider, B. Hof, and J. Westerweel, Annu. Rev. Fluid Mech. 39, 447 (2007).

[26] K. O. Mikaelian, Phys. Rev. A 33, 1216 (1986).

[27] R. H. Kraichnan, Phys. Fluids 5, 1374 (1962).

[28] G. Ahlers, S. Grossmann, and D. Lohse, Rev. Mod. Phys. 81, 503 (2009).

[29] G. Boffetta, A. Mazzino, S. Musacchio, and L. Vozella, Phys. Rev. E 79, 065301 (2009).

[30] J. J. Niemela and R. J. Donnelly, Phys. Rev. Lett. 59, 2431 (1987).

[31] A. Swaminathan, Ph.D. thesis, The Pennsylvania State University, 2017. 